\title{
An Approach to Overcome the Uneven Shade Problem of Compact Knitted Fabric with Reactive Turquoise Dye
}

\author{
Muhammad Abdur Rashid", Md. Delwar Hossain \\ Department of Textile Engineering, Dhaka University of Engineering \& Technology, Bangladesh
}

Copyright (C) 2015 by authors, all rights reserved. Authors agree that this article remains permanently open access under the terms of the Creative Commons Attribution License 4.0 International License

\begin{abstract}
Reactive colors such as turquoise have definite properties of larger structure results uneven shade problem for fabric with compact structure. In this research the uneven shade problem was resolved by using stripping process in the preparatory stage before dyeing with the help of Hydrose $\left(\mathrm{Na}_{2} \mathrm{~S}_{2} \mathrm{O}_{4}\right)$ treatment. Various test such as fabric strength, color fastness, color strength have been carried out to study the performance of experimental process. The obtained results shows that, stripping process at preparatory stage reflect significant effect on turquoise dye for even dye shade. As a result uneven shade will not be visualized due to the uniformly absorption of dye molecules into the fiber core. The morphological changes of the fiber in dyed fabric were studied by using Scanning Electron Microscope (SEM).
\end{abstract}

Keywords Turquoise Reactive Dye, Hydrose, Uneven Shade, Compact Knitted Fabric

\section{Introduction}

Since knit dyeing process is one of the most important sectors in Bangladesh this is because Bangladesh earns around $40 \%$ of its foreign currency by this sector [1]. Cotton is the most important natural textile fiber and used to produce apparel, home furnishings, and industrial products [2]. Most of the dyes do not chemically react with the cellulose molecule to affix the color. True chemical reaction between cellulose and the dye molecule occurs with reactive dyes due to the presence of hydroxyl group of cellulose and functional group of dye molecules [3]. But some of reactive dyes such as turquoise have definite properties which does not mostly yield even shade with the single jersey cotton fabric which GSM ranges from 120 to 160 [3]. Higher compactness of the fabric structure, dye molecules could not penetrate into the core of cotton fibers. When stripping was done of the fabric, compactness was reduced as well as size of the porosity of cotton fiber might be large due to the action of hydrose. The main function of the hydrose is not only to remove color from reactive dyed fabric but also it could be reduced the compactness and increased size of the porous of cotton fibers. Fabrics having quite relatively compact structure that does not allow proper penetration of dye molecules evenly into fiber polymer system. Due to those problems, the knit dyeing factories in Bangladesh have been facing a lot of problems. In present days, these factories are practicing re-dyeing like topping and stripping and dyeing. As a result, production rate becomes lower, fabrics damage, production cost, chemicals and water consumption become higher, as a result pollutants generation goes higher. To solve this problem, the single jersey compact fabric may be treated in different manner in the preparatory stage. The conventional pretreatment process involved scouring, bleaching before dyeing [4]. But at present research this problem can be solved by using stripping process at preparatory stage and reduce uneven shade problem during coloration of turquoise color of reactive dye.

\section{Materials and Methods}

\subsection{Materials}

The investigation has been carried out with $100 \%$ cotton knitted compact single jersey fabric. The yarn count was $24^{\mathrm{s}} / 1$ carded yarn, the fabric weight per unit area was 150 $\mathrm{g} / \mathrm{m}^{2}$, course density was 12 courses/cm and wales's density was 11 wales $/ \mathrm{cm}$. The fabric was scoured and stripped by caustic soda and hydrose in a single bath stage with the standard recipe. Then the knitted compact fabrics were dyed with turquoise reactive dye in darker shade percentage.

\subsection{Methods}

\subsubsection{Scouring}

Scouring was performed to remove any impurities present in the fabric. The impurities i.e. oil \& wax, lubricants, dirt, surfactants, residual tints etc [5] are removed using an alkaline solution, typically sodium hydroxide, at high temperatures to breakdown or emulsify and saponify 
impurities [6].

\subsubsection{Stripping}

The scoured fabric was performed stripping (IR lab dyeing machine, sandolab, supermat, copower technology co. ltd. Taiwan) according to the following suitable recipe (Table 1) with maintain proper time and temperature. After that, the sample was neutralized by $1 \mathrm{gm} / 1$ acetic acid at $60^{\circ} \mathrm{C}$ for 10 minutes and then performed cold wash.

Table 1. Recipe of stripping process

\begin{tabular}{|c|c|}
\hline Process parameter & Amount \\
\hline Hydrose & $: 6 \mathrm{gm} / 1$ (Dosing at $80^{\circ} \mathrm{C}$ for $10 \mathrm{~min}$ ) \\
\hline Sodium hydroxide & $: 3 \mathrm{gm} / 1$ (Dosing at $\left.60^{\circ} \mathrm{C} 10 \mathrm{~min}\right)$ \\
\hline Sequestering agent & $: 0.5 \mathrm{gm} / 1$ \\
\hline Wetting agent & $: 1 \mathrm{gm} / 1$ \\
\hline Temperature & $: 80^{\circ} \mathrm{C}$ \\
\hline Time & $: 30 \mathrm{~min}$ \\
\hline $\mathrm{M}: \mathrm{L}$ & $: 1: 10$ \\
\hline
\end{tabular}

\subsubsection{Dyeing}

The scoured and stripping knitted fabric was dyed (IR lab dyeing machine, sandolab, supermat, copower technology co. ltd. Taiwan) by combined shade $(3.22 \%)$ according to the following suitable recipe(Table 2) with proper process parameters [7]. Then the dyed samples were neutralized by $1.5 \mathrm{gm} / 1$ acetic acid at $40^{\circ} \mathrm{C}$ for 15 minutes. After that hot wash was performed by $1 \mathrm{gm} / 1$ detergent at $90^{\circ} \mathrm{C}$ for 20 minutes and then followed cold wash.

Table 2. Recipe of dyeing process for compact knitted fabric

\begin{tabular}{|c|c|}
\hline Process parameter & Amount \\
\hline GSS (Leveling agent) & $: 0.12 \mathrm{gm} / 1$ \\
\hline Arboquest 340 (Sequester) & $: 1 \mathrm{gm} / 1$ \\
\hline Albafluid C (Anti-creasing) & $: 1 \mathrm{gm} / 1$ \\
\hline Glauber salt & $: 35 \mathrm{gm} / 1$ \\
\hline Reactive Turquoise Blue H2GP & $: 2.52 \%$ \\
\hline Reatobond Blue RR & $: 0.06 \%$ \\
\hline Indofix yellow ME4GL & $: 0.64 \%$ \\
\hline Sodium carbonate & $: 15 \mathrm{gm} / 1\left(\mathrm{at} 50^{\circ} \mathrm{C}\right.$ for $\left.20 \mathrm{~min}.\right)$ \\
\hline Time & $: 30 \mathrm{~min}$ \\
\hline Temperature & $: 60^{\circ} \mathrm{C}$ \\
\hline M:L & $: 1: 10$ \\
\hline
\end{tabular}

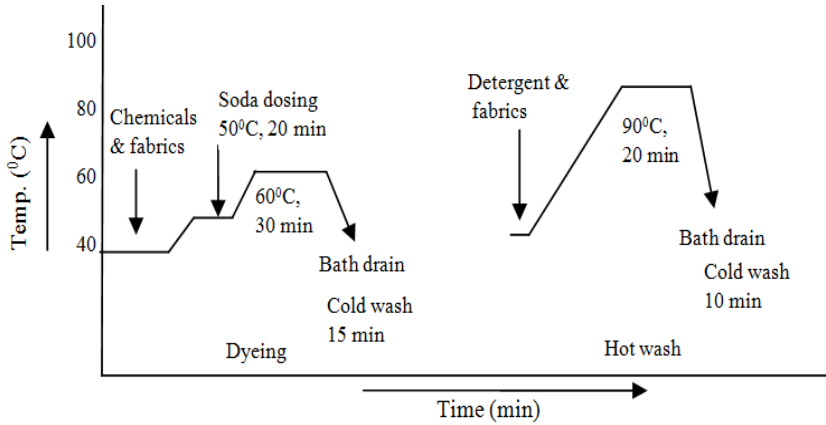

Figure 1. Process curve for compact knitted fabric dyeing

2.2.4. Characterizing the dyed compact cotton knitted fabric

At first treated all dyed cotton knitted fabric were conditioned in $65 \% \mathrm{RH}$ and at $20^{\circ} \mathrm{C}$ for 24 hours before testing according to ASTM D1776 [8]. Then bursting strength of the dyed cotton knitted fabric was determined according to ASTM D 3787 [9] (Ball Burst test). The values for color fastness were rated with a Grey scale for color change according to AATCC test method 61 [10]. SEM was studied using a scanning electron microscopy. Dye yield capacities $(\mathrm{K} / \mathrm{S}), \mathrm{CMC}$ value of the fabrics were measured by using Data color spectrophotometer according to ASTM D 1925 [11].

\section{Results and Discussions}

\subsection{Measurement of Color Strength}

The values of K/S for the dyed samples were usually a linear function of the amounts of colorants present in the sample, since most of the dyes presented in the fabric did not contribute significantly to light scattering. The following figure 2 shows the K/S values of the dyed samples. The K/S value of dyed fabric after stripping was more than the normal processing fabric. The possible explanation could be the fabric was more swelling due to the stripping process.

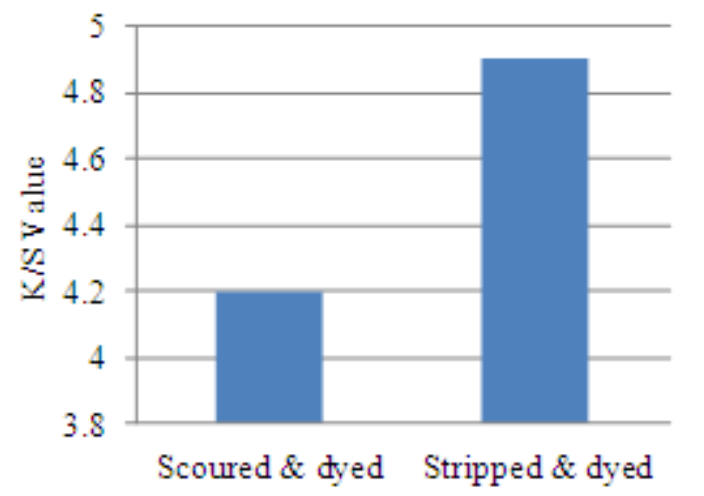

Figure 2. K/S value of scoured and stripped dyed fabric 
Table 3. Color difference value of stripped dyed and scoured dyed fabric

\begin{tabular}{|c|c|c|c|c|c|c|c|c|}
\hline Sample & Sample No. & $\mathrm{Ill} / \mathrm{Obs}$ & DL* & $\mathrm{Da}^{*}$ & $\mathrm{Db}^{*}$ & $\mathrm{DC}^{*}$ & $\mathrm{DH}^{*}$ & $\mathrm{CMC}$ \\
\hline \multirow{2}{*}{$\begin{array}{l}\text { Stripped dyed to scoured } \\
\text { dyed fabric }\end{array}$} & Sample 1 & \multirow{2}{*}{ D65 $10^{\circ}$} & 2.53 & 1.82 & -0.37 & -1.80 & 0.44 & 1.36 \\
\hline & Sample 2 & & 2.38 & 1.43 & -0.83 & -1.39 & 0.90 & 1.29 \\
\hline
\end{tabular}

\subsection{Measurement of Color Difference Value}

In the comparison between stripped dyed and scoured dyed samples gave CMC value 1.36 (sample 1) and 1.29 (sample 2) in table 3, that express fail because their dye molecules absorption were difference. The allowable explanation could be the stripped fabric become more effective because during stripping process, the samples were more swelling and absorbed more dye molecules. On the other hand only scoured sample was less effective due to the less swelling of the sample and absorbed less dye molecules.

\subsection{Measurement of Fabric Strength}

Comparison strength among grey, scoured, stripped, scoured \& dyed and stripped \& dyed knitted fabric has been done and the experimental results in Figure 3 shows maximum strength could be attained in the scoured white fabric because higher moisture might be retained than the grey fabric in normal atmospheric condition with compared to mercerization effects due to the increasing hydrogen bonds. Minimum strength might be shown in the stripped \& scoured dyed fabric due to the deterioration of polymer chain.

\subsection{Assessment of Color Fastness}

In this paper has been carried out various types of fastness properties of dyed fabric such as color fastness to wash, color fastness to perspiration and color fastness to rubbing.

\subsubsection{Color fastness to wash}

There was a little difference in wash fastness test between the dyed samples that have been dyed after scouring and stripping. This may be occurred due to dye molecules that are loosely attached on the fabric surface that have not been formed any bonds with the fiber polymer system.

\subsubsection{Color fastness to perspiration}

In this experiment color fastness to perspiration was evaluated for stripped dyed and scoured dyed fabric. Both processes gave almost similar result due to the similar bonding of dyes and fibers.

\subsubsection{Color fastness to rubbing}

In the paper color fastness to rubbing assessments are done for both fabrics dyed before scouring and stripping. This test was designed to determine the degree of color which may be transferred from the surface of a colored fabric to a specific test cloth for rubbing which could be dry and wet. This test result showed in Figure 6 where better rubbing fastness for stripped dyeing compared to scoured dyeing fabric. Because there may be a better dye-fiber bond than the latter process for it's more porous structure that may allow the dye-fiber bonding.

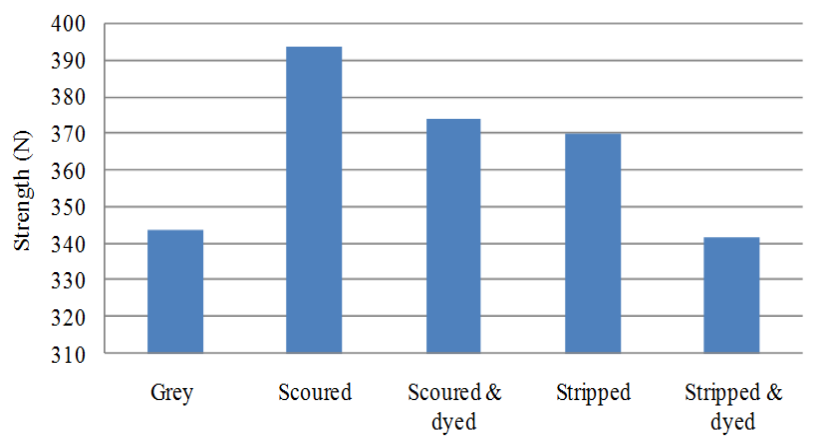

Figure 3 Strength of grey and different processing fabric

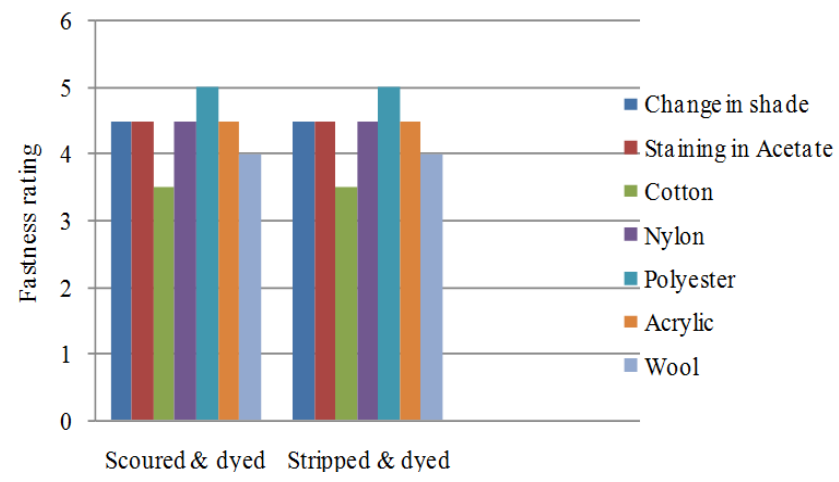

Figure 4. Color fastness to wash rating of scoured and stripped dyed fabric

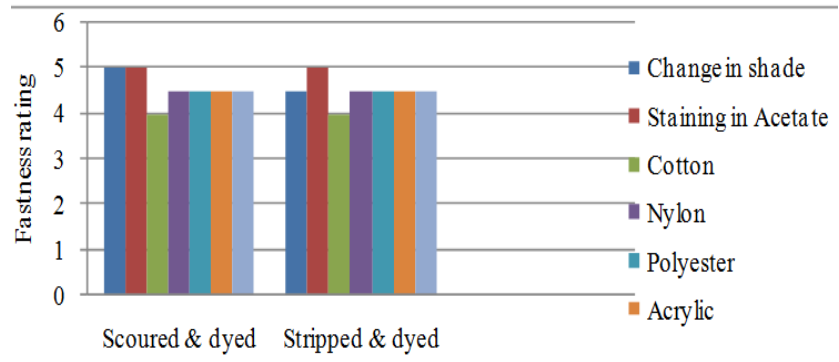

Figure 5. Color fastness to perspiration rating of scoured and stripped dyed fabric 

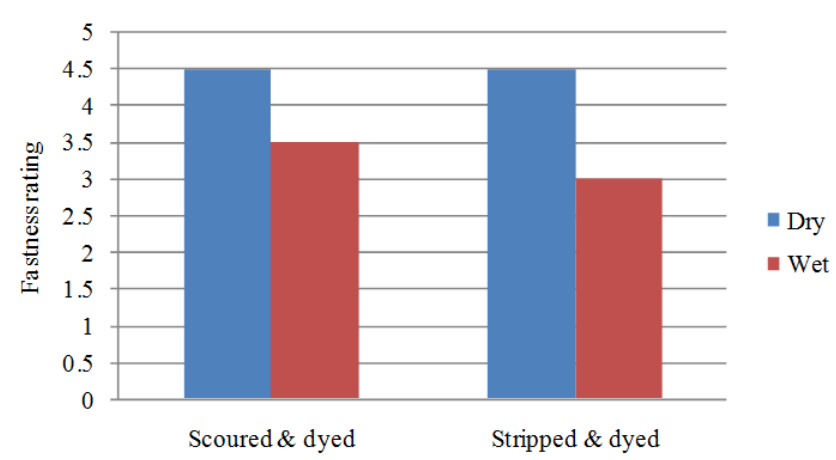

Figure 6. Color fastness to rubbing rating of scoured and stripped dyed fabric

\subsection{Microstructure Analysis of Treated Samples}

The SEM result of scoured dyed fabric showed bright appears due to its compactness and finer cross-section (5.6 $\mu \mathrm{m}, 10.1 \mu \mathrm{m}, 12.8 \mu \mathrm{m}, 16.2 \mu \mathrm{m}$ etc.). So it was found the smaller size porosity than the stripped dyed fabric. That may limit the dye molecules absorption by the fabric. But stripped dyed fabric absorb more light due to the looseness of fabric structure and coarser cross-section $(9.43 \mu \mathrm{m}, 14.2 \mu \mathrm{m}, 19.3$ $\mu \mathrm{m}, 21.7 \mu \mathrm{m}$ etc.) was found that occupied larger size porosity on its surface which might allow easily penetration of lager size dye molecules that may reduce the uneven shade problem.

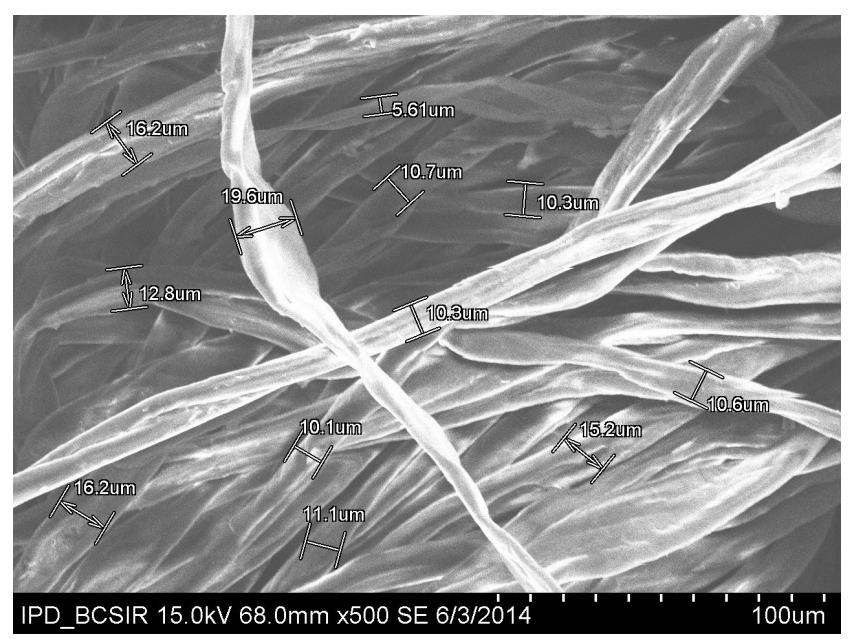

Figure 7. SEM representation of the scoured dyed fabric

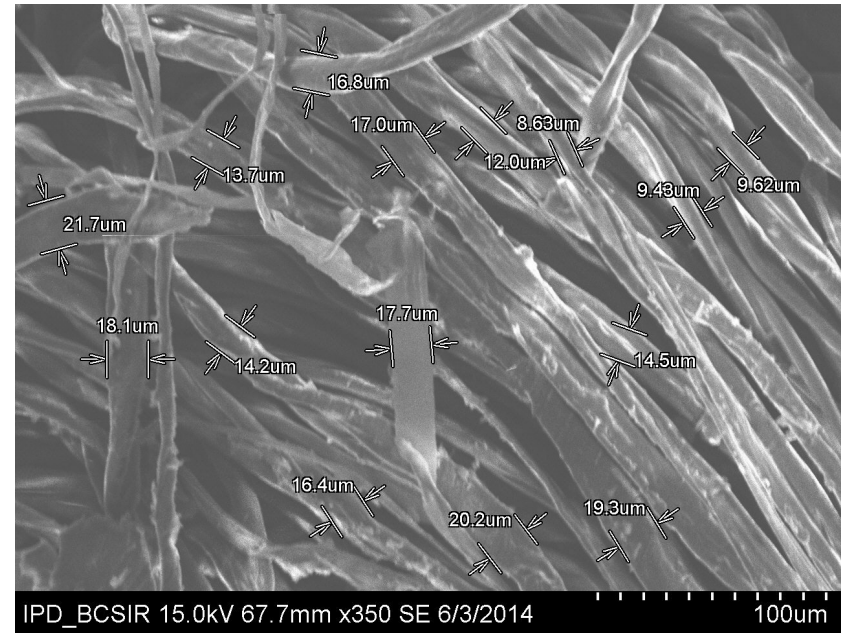

Figure 8. SEM representation of the stripped dyed fabric

\section{Conclusions}

The study was found that grey fabric stripping reduced in uneven shade in case of compact single jersey knit fabric when it was dyed with turquoise color of reactive dye. On the other hand conventional dyeing process gives uneven shade and need to be stripped after dyeing and re-dyeing to match the shade with losing fabric strength and other fabric quality. Different types of physical quality tests also exhibited that the stripping dyed process gave better result than other processes. The wash fastness and rubbing fastness tests of different dyed samples may not be revealed significant difference. In the comparison of stripped dyed fabric with the scoured dyed fabric would be showed CMC value 1.36 (sample 1) and 1.29 (sample 2) due to non-uniform absorbency of dye molecules. In case of SEM stripped dyed fabric would be shown larger porous on the cotton fabric surface due to the action of hydrose but scoured dyed fabric would not be shown larger porous on the cotton fabric surface due to the absence of action of hydrose because partial breakage of hydrogen bonds. As a result larger turquoise color dye molecules could be penetrated more evenly into the cotton fiber core of the stripped fabric which might help to reduce uneven shade problem in case compact single jersey knit fabric. 


\section{REFERENCES}

[1] Data Source: Export Promotion Bureau Complied by BGMEAhttp://bgmea.com.bd/home/pges/TradeInormation\#. UxYBcOIpfIU

[2] Cotton for Nonwovens, "A Technical Guide", Cotton Incorporated.

[3] Shore, John, "Cellulosics Dyeing", Society of Dyers and Colorist, England, 1995, p189-241

[4] Trotman, E.R, M.B.E., "Dyeing and chemical technology of textile fibers", London, Fourth edition.

[5] Hossain, Md Delwar, Rashid, Muhammad Abdur, Kafi, Md Abdullahil, and Sarker, Forkan, "Investigation on Physical Properties of Fluorescent Dyed Cotton Knit Fabric", Chemical and Materials Engineering, Vol. 2, No 5, 2014, p. 101-105.

[6] Tomasino, Charles, "Chemistry \& technology of fabric preparation \& finishing", NC: North Carolina State University, 1992.
[7] Rashid, Muhammad Abdur, Hossain, Md Delwar, Kafi, Md Abdullahil, and Yesmin, Sabina, "Process Modification of Denim Garments Dyeing with Fluorescent Dye," International Journal of Textile Science Vol. 3, No. 2, 2014, p. 33-37.

[8] ASTM D 1776, "Standard practice for conditioning textiles for testing," American Society for Testing and Materials, Annual Book of ASTM Standards, vol. 7(1). ASTM International, West Conshohocken, PA, USA, 2008.

[9] ASTM D5034, "Standard test method for breaking force and elongation of textile fabrics (Grab test)," American Society for Testing and Materials, Annual book of ASTM Standards, 7(1), ASTM International, West Conshohocken, PA, USA, 2009.

[10] AATCC test method 61, "Colorfastness to laundering, home and commercial: accelerated," American Association of Tex Research Triangle Park, N.C., USA, 2010.

[11] AATCC test method 170, "Calculation of small color differences for acceptability," American Association of Tex Research Triangle Park, N.C., USA, 2009 